\title{
QUANTIFICAÇÃO DE BIOMASSA E ESTIMATIVA DE ESTOQUE DE CARBONO EM UMA CAPOEIRA DA ZONA DA MATA MINEIRA ${ }^{1}$
}

\author{
Sabina Cerruto Ribeiro², Laércio Antônio Gonçalves Jacovine ${ }^{3}$, Carlos Pedro Boechat Soares ${ }^{3}$, \\ Sebastião Venâncio Martins ${ }^{3}$, Áurea Maria Brandi Nardelli ${ }^{4}$ e Agostinho Lopes de Souza ${ }^{3}$
}

\begin{abstract}
RESUMO - Este estudo teve como objetivo quantificar os estoques de volume, de biomassa total com casca e de carbono em um fragmento de Floresta Estacional Semidecidual em estádio secundário médio (capoeira) na Zona da Mata mineira, no Município de Viçosa, MG. Para a conversão de volume em biomassa foram obtidas estimativas de densidade básica das 10 espécies de maior valor de importância (VI). O estoque de carbono foi determinado considerando-se que a biomassa seca contém cerca de $50 \%$ de carbono. Foram contabilizadas 31 espécies arbóreas, distribuídas em 29 gêneros e 21 famílias. A biomassa total média das árvores foi de 38,99 t.ha-1 o que correspondeu a um estoque de carbono de 19,50 \pm 8,08 tC. ha ${ }^{-1}$. O valor encontrado foi considerado baixo quando comparado com o de outros estudos. Uma explicação para isso podem ser as influências sofridas pelo uso anterior da área e a ação de efeitos de borda na capoeira, o que contribuiu para a menor estocagem de biomassa e de carbono. As estimativas obtidas para a capoeira podem ser usadas como subsídios para a elaboração de projetos de florestamento/reflorestamento do Mecanismo de Desenvolvimento Limpo.
\end{abstract}

Palavras-chave: Biomassa, Estoque de carbono e Mata Atlântica.

\section{QUANTIFICATION OF BIOMASS AND ESTIMATION OF CARBON STOCK IN A CAPOEIRA IN THE MINAS GERAIS FOREST ZONE}

\begin{abstract}
The present study aimed to quantify the stocks of volume, total biomass with bark and carbon in a fragment of Semideciduous Seasonal Forest in mid-secondary succession stage (capoeira) located in the Minas Gerais forest zone, in Viçosa, the State of Minas Gerais, Brazil. For the volume conversion in biomass, estimates of basic density of 10 species with higher IV (Importance Value) were obtained. The stock of carbon was determined considering that the dry biomass contains, approximately, 50\% carbon. 31 tree species were registered, distributed in 29 genera and 21 families. The trees' average total biomass was 38.99 t.ha $^{-1}$, which corresponded to a carbon stock of $19.50 \pm 8.08 \mathrm{tC} . \mathrm{ha}^{-1}$. The value found is considered low when compared to other studies. Influences of prior use and edge effects may explain the low biomass and carbon stock found. The estimates obtained for the capoeira can be used as subsidies for the elaboration of afforestation/reforestation projects in the Clean Development Mechanism.
\end{abstract}

Keywords: Biomass, Carbon stock and Atlantic Forest.

\section{INTRODUÇÃO}

O aumento da concentração de gases de efeito estufa na atmosfera terrestre provenientes de atividades antrópicas tem levado à intensificação do efeito estufa natural e, em consequência, às mudanças climáticas globais. Em virtude desse panorama, em 1997 foi elaborado o Protocolo de Quioto, que entrou em vigor em fevereiro de 2005.
Esse Protocolo prevê que as emissões de gases de efeito-estufa, no período de 2008 a 2012, sejam reduzidas, em média, a 5,2\% abaixo dos níveis de 1990 (ano de inventário dos gases). Objetivando facilitar o cumprimento das metas de redução dessas emissões, o Protocolo de Quioto trouxe como inovação os seguintes mecanismos de flexibilização: Comércio de Emissões, Implementação Conjunta e Mecanismo de

\footnotetext{
${ }^{1}$ Recebido em 25.05.2008 e aceito para publicação em 02.03.2010.

${ }^{2}$ Programa de Pós-Graduação em Ciência Florestal pela Universidade Federal de Viçosa, Brasil. E-mail: <sabina_ribeiro@yahoo.com.br> . ${ }^{3}$ Universidade Federal de Viçosa - Brasil.E-mail: <jacovine@ufv.br>, <csoares@ufv.br>, <venancio@ufv.br>e <alsouza@ufv.br>.

${ }^{4}$ Auditora e revisora técnica do programa SGS Climate Change. E-mail: <aurea_nardelli@yahoo.com.br>.
} 
Desenvolvimento Limpo (MCT, 2001). Entre eles, apenas o Mecanismo de Desenvolvimento Limpo (MDL) permite a participação de países em desenvolvimento, como o Brasil, e assim, a geração de créditos de carbono por essas nações.

Nesse contexto, as florestas têm sua importância destacada graças à capacidade natural dos vegetais de sequestrar $\mathrm{CO}_{2}$ da atmosfera, por meio da fotossíntese, e fixá-lo sob a forma de carbono na matéria lenhosa (CHANG, 2004), o que corrobora com a sua inclusão em projetos de remoções de emissões no MDL. Entretanto, a fim de que se possa avaliar o potencial para a geração de créditos de carbono em florestas é de fundamental importância que sejam obtidas estimativas confiáveis do estoque de carbono presente na biomassa da vegetação. Dessa forma, é possível estimar a quantidade de carbono, emitida ou sequestrada, no tempo e no espaço (HIGUCHI et al., 2004).

Considerando a carência de estudos sobre a fixação de carbono em florestas nativas pertencentes ao Bioma Mata Atlântica, objetivou-se, com este trabalho, quantificar a biomassa total com casca e o estoque de carbono em árvores de uma capoeira de um fragmento florestal da Zona da Mata no Município de Viçosa, Minas Gerais.

\section{MATERIAL E MÉTODOS}

O estudo foi desenvolvido no Sítio Bom Sucesso, localizadonomunicípio de Viçosa (2045’23”S e 4252’23”W), na Zona da Mata do estado de Minas Gerais. O fragmento florestal, onde foram executadas as coletas de dados é conhecido na região como "Mata do seu Nico" e engloba uma área de aproximadamente 35 ha.

A “Mata do seu Nico” é considerada um dos fragmentos florestais mais bem conservados da região. Divide-se em duas partes, com características bem diversas: floresta primária e capoeira. A capoeira, onde os dados foram coletados, possui cerca de 30 anos. Essa área sofreu intervenção antrópica, com o estabelecimento de pastagem no local, conforme informações dos proprietários. Depois de finalizado esse uso, a área foi abandonada e a regeneração teve início. Atualmente a capoeira tem a presença de bovinos como a sua principal forma de distúrbio.

Os solos predominantes da região são o Latossolo Vermelho-Amarelo álico nos topos de morros e nas encostas e o Podzólico Vermelho-Amarelo câmbico nos terraços (REZENDE, 1971; CORRÊA, 1984). Segundo Silva et al. (2003), a altitude da região é de 670 m, com relevo variando de ondulado a montanhoso. A área de estudo pertence à formação Floresta Estacional Semidecidual Montana (VELOSO et al., 1991).

O clima da região, segundo a classificação de Köppen, é Cwa, mesotérmico úmido, com verões chuvosos e invernos secos. A precipitação pluvial média anual é de $1.248 \mathrm{~mm}$, a umidade relativa do ar média anual é de 80,6\% (SOARES JÚNIOR, 2000) e a temperatura média anual é de $21^{\circ} \mathrm{C}$ (SILVA et al., 2003 apud CASTELLANI, 2006).

A caracterização das principais espécies florestais presentes na capoeira foi feita com base no estudo de Pinto (2005), o qual foi desenvolvido em um fragmento próximo e com características semelhantes às da capoeira da "Mata do seu Nico". A confirmação e atualização dos nomes científicos e das famílias foram feitas com base no site do Missouri Botanical Garden (MOBOT, 2007).

Do estudo de Pinto (2005) foram obtidos dados de densidade absoluta (DA), densidade relativa (DR), frequência absoluta (FA), frequência relativa (FR), dominância absoluta (DoA), dominância relativa (DoR) e valor de importância (VI), além da listagem de espécies (Tabela 1).

Para a coleta de dados quantitativos foi utilizada a distribuição sistemática de linhas de amostragem e de distância entre as parcelas, a fim de minimizar ao máximo os efeitos de borda. No entanto, devido à presença de obstáculos naturais, algumas linhas sofreram pequeno ajuste em relação ao local pré-definido. Dessa forma, foram estabelecidas duas linhas de amostragem, com cinco parcelas de $10 \mathrm{~m}$ x $50 \mathrm{~m}$ cada e distância entre linhas de aproximadamente $33 \mathrm{~m}$ (Figura 1). As medições em cada parcela abrangeram a altura total $\left(\mathrm{H}_{\mathrm{t}}\right)$ e a Circunferência à Altura do Peito (CAP) das árvores com CAP $\geq 15,7$ cm (DAP, Diâmetro à Altura do Peito $\geq 5 \mathrm{~cm}$ ).

Em cada parcela da capoeira foram calculados os seguintes parâmetros populacionais: número de indivíduos por hectare (N/ha), área basal por hectare (AB/ha), volume total por hectare (Vt/ha), diâmetro médio (q) e altura total média ( $\bar{H}$ ), conforme os estimadores apresentados no Tabela 2. Além disso, para cada parâmetro foram atribuídos os valores mínimo e máximo, assim como os valores referentes à média e ao desvio-padrão. 
Tabela 1 - Parâmetros da estrutura horizontal da capoeira, segundo Pinto (2005), ordenados decrescentemente pelo valor de importância (VI\%): densidade absoluta (DA), densidade relativa (DR), frequência absoluta (FA), frequência relativa (FR), dominância absoluta (DoA), dominância relativa (DoR) e valor de cobertura (VC\%).

Table 1 - Horizontal structure parameters of capoeira, according to Pinto (2005), showing a decreasing order of Importance Value (VI\%): absolute density (DA), relative density (DR), absolute frequency (FA), relative frequency (FR), absolute dominance (DoA), relative dominance (DoR) and covering value (VC).

\begin{tabular}{|c|c|c|c|c|c|c|c|c|}
\hline Espécies & DA & DR & FA & FR & DoA & DoR & VI & VC \\
\hline Piptadenia gonoacantha (Mart.) J. F. Macbr. & 160,0 & 12,03 & 100,00 & 5,56 & 54,64 & 29,70 & 15,76 & 20,87 \\
\hline Vernonanthura diffusa (Less.) H. Rob. & 146,7 & 11,03 & 80,00 & 4,44 & 13,73 & 7,46 & 7,64 & 9,25 \\
\hline Miconia cinnamomifolia (DC.) Naudin & 90,0 & 6,77 & 90,00 & 5,00 & 20,48 & 11,10 & 7,62 & 8,94 \\
\hline Piptocarpha macropoda (DC.) Baker & 100,0 & 7,52 & 70,00 & 3,89 & 17,49 & 9,51 & 6,97 & 8,52 \\
\hline Luehea grandiflora Mart. & 36,7 & 2,76 & 50,00 & 2,78 & 16,86 & 9,17 & 4,90 & 5,97 \\
\hline $\begin{array}{l}\text { Senna macranthera (DC. ex Collad.) H.S. Irwin } \\
\text { \& Barneby }\end{array}$ & 56,7 & 4,26 & 80,00 & 4,44 & 0,57 & 3,09 & 3,93 & 3,68 \\
\hline Siparuna guianensis Aubl. & 70,0 & 5,26 & 90,00 & 5,00 & 0,22 & 1,20 & 3,82 & 3,23 \\
\hline Sparattosperma leucanthum (Vell.) K. Schum. & 56,7 & 4,26 & 70,00 & 3,89 & 0,56 & 3,06 & 3,74 & 3,66 \\
\hline Xylopia sericea A. St.-Hil. & 33,3 & 2,51 & 60,00 & 3,33 & 0,22 & 1,21 & 2,35 & 1,86 \\
\hline Trichilia pallida Sw. & 43,3 & 3,26 & 50,00 & 2,78 & 0,12 & 0,67 & 2,24 & 1,97 \\
\hline Erythroxylum pelleterianum A. St.-Hil. & 33,3 & 2,51 & 50,00 & 2,78 & 0,11 & 0,59 & 1,96 & 1,55 \\
\hline Cecropia hololeuca Miq. & 10,0 & 0,75 & 30,00 & 1,67 & 0,45 & 2,47 & 1,63 & 1,61 \\
\hline Myrcia fallax (Rich.) DC. & 16,7 & 1,25 & 50,00 & 2,78 & 0,14 & 0,77 & 1,60 & 1,01 \\
\hline Zanthoxylum rhoifolium Lam & 23,3 & 1,75 & 40,00 & 2,22 & 0,15 & 0,82 & 1,60 & 1,29 \\
\hline Vismia guianensis (Aubl.) Pers. & 20,0 & 1,50 & 50,00 & 2,78 & 0,09 & 0,47 & 1,58 & 0,99 \\
\hline Cecropia glaziovi Snethl. & 20,0 & 1,50 & 40,00 & 2,22 & 0,13 & 0,72 & 1,48 & 1,11 \\
\hline Lacistema pubescens Mart. & 13,3 & 1,00 & 30,00 & 1,67 & 0,05 & 0,26 & 0,98 & 0,63 \\
\hline Protium warmingiana March, L. & 13,3 & 1,00 & 30,00 & 1,67 & 0,05 & 0,26 & 0,98 & 0,63 \\
\hline Myrcia rostrata DC. & 10,0 & 0,75 & 30,00 & 1,67 & 0,04 & 0,19 & 0,87 & 0,47 \\
\hline Matayba elaeagnoides Radlk. & 10,0 & 0,75 & 30,00 & 1,67 & 0,03 & 0,15 & 0,86 & 0,45 \\
\hline Casearia sylvestris Sw. & 6,7 & 0,50 & 20,00 & 1,11 & 0,06 & 0,35 & 0,65 & 0,43 \\
\hline Sapium glandulatum (Vell.) Pax & 6,7 & 0,50 & 20,00 & 1,11 & 0,05 & 0,29 & 0,63 & 0,40 \\
\hline Dictyoloma vandellianum A.H.L. Juss. & 6,7 & 0,50 & 20,00 & 1,11 & 0,05 & 0,26 & 0,62 & 0,38 \\
\hline $\begin{array}{l}\text { Schefflera morototoni (Aubl.) Maguire, Steyerm. } \\
\text { \& Frodin }\end{array}$ & 6,7 & 0,50 & 20,00 & 1,11 & 0,03 & 0,14 & 0,58 & 0,32 \\
\hline Dalbergia nigra (Vell.) Allemao ex Benth. & 6,7 & 0,50 & 10,00 & 0,56 & 0,04 & 0,20 & 0,42 & 0,35 \\
\hline $\begin{array}{l}\text { Sorocea bonplandii (Baill.) W.C. Burger, Lanj. } \\
\text { \& Wess. Boer }\end{array}$ & 3,3 & 0,25 & 10,00 & 0,56 & 0,04 & 0,24 & 0,35 & 0,25 \\
\hline Alchornea glandulosa Poepp. & 3,3 & 0,25 & 10,00 & 0,56 & 0,03 & 0,18 & 0,33 & 0,22 \\
\hline Bauhinia forficata Link & 3,3 & 0,25 & 10,00 & 0,56 & 0,01 & 0,08 & 0,30 & 0,17 \\
\hline Croton floribundus Spreng. & 3,3 & 0,25 & 10,00 & 0,56 & 0,01 & 0,04 & 0,28 & 0,15 \\
\hline Machaerium nyctitans (Vell.) Benth. & 3,3 & 0,25 & 10,00 & 0,56 & 0,01 & 0,04 & 0,28 & 0,15 \\
\hline Solanum cernuum Vell. & 3,3 & 0,25 & 10,00 & 0,56 & 0,01 & 0,04 & 0,28 & 0,15 \\
\hline
\end{tabular}

O volume total com casca de árvores individuais, no estádio sucessional da capoeira, foi obtido por meio da equação $1\left(\mathrm{r}_{\mathrm{yw}}=0,973\right)$, conforme CETEC (1995):

$$
V t_{c}=0,000074 * D A P^{1,707348} * H t^{1,16873}
$$

em que: $\mathrm{em} \mathrm{m}^{3}$;

$V t_{c}=$ volume total com casca de árvores individuais,

$D A P=$ diâmetro à altura do peito, em cm; e

$H_{t}=$ altura total, em $\mathrm{m}$.
Em virtude de restrições de ordens legal e operacional, usou-se o método não destrutivo, pelo qual a estimativa da biomassa é feita a partir do volume e densidade básica média da madeira.

A seleção das espécies consideradas representativas na capoeira foi feita com base nos dados de densidade relativa (DR) e valor de importância (VI), determinados por Pinto (2005). Em seguida, para cada espécie selecionada foi atribuído o valor de densidade básica da madeira (LORENZI, 2002a; LORENZI, 2002b; OLIVEIRA et al., 2005; PAULA et al., 1995; STEEGE et al., 2000). Como a densidade básica da madeira da 
Fonte: Adaptado de NEPUT.

Source: Adapted from NEPUT.

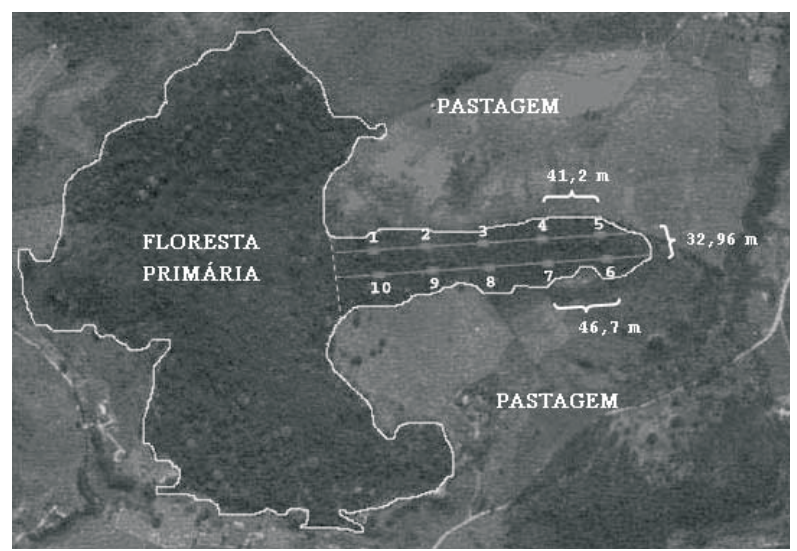

Figura 1 - Posicionamento das parcelas na capoeira (escala $1: 15000)$.

Figure 1 - Plot's location at capoeira (scale 1:15000).

Tabela 2-Variáveis estimadas nos parâmetros populacionais e respectivos estimadores.

Table 2 - Estimated variables in the population parameters and respective estimators.

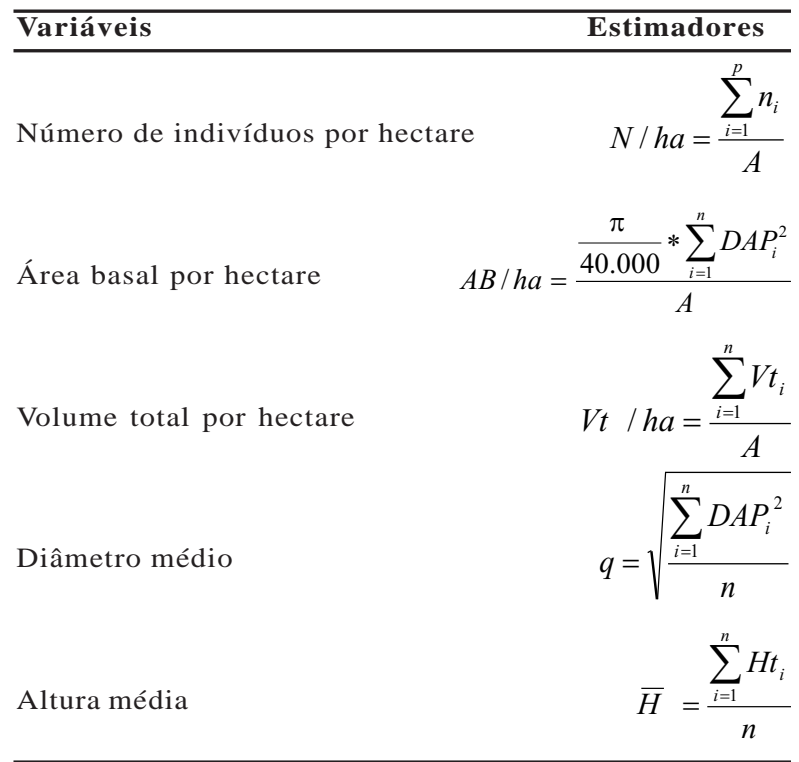

Em que: $\mathrm{n}$ = número de indivíduos medidos em cada parcela; p = número da parcela; $\mathrm{A}$ = área da parcela, correspondendo a 0,05 ha; $\mathrm{DAP}_{\mathrm{i}}=$ diâmetro com casca da i-ésima árvore medida a $1,30 \mathrm{~m}$ do solo, em $\mathrm{cm} ; \mathrm{Vt}=$ volume total com casca da i-ésima árvore qualificada estimado por equação de volume de árvore individual (CETEC, 1995), $\mathrm{m}^{3}$; e $\bar{H}=$ altura total da i-ésima árvore qualificada, em $\mathrm{m}$.

Revista Árvore, Viçosa-MG, v.34, n.3, p.495-504, 2010 espécie Vernonanthura diffusa não estava disponível na literatura, ela foi determinada em laboratório, por meio da norma NBR 11941 (ABNT, 2003).

Com base nos valores de densidade básica convertidos em $\mathrm{kg} . \mathrm{m}^{-3}$, foi calculado um valor de densidade básica média $(\bar{d})$ para a capoeira, ponderada pelo valor de cobertura e calculado com base no estudo de Pinto (2005), conforme a equação 2.

em que:

$$
\bar{d}=\sum_{i=1}^{n} D b_{i} *\left(\frac{V C_{i}}{\sum_{i=1}^{n} V C_{i}}\right)
$$

$n$ = número de espécies selecionadas;

$D b_{i}=$ densidade básica da i-ésima espécie selecionada, em kg.m ${ }^{-3}$; e

$V C_{i}=$ média do valor de cobertura das i-ésimas espécies selecionadas, em porcentagem.

A seguir, estimou-se a biomassa da madeira de cada árvore pela multiplicação da densidade básica média da madeira pelos volumes totais com casca (equação 1), conforme a equação 3.

em que:

$$
B=\bar{d} * \hat{V} t_{c}
$$

$B$ = biomassa da árvore, em $\mathrm{kg}$;

$\bar{d}=$ densidade básica média, em kg. $\mathrm{m}^{-3}$; e

$\hat{V} t_{c}=$ volume total com casca estimado de árvores individuais, em $\mathrm{m}^{3}$.

A estimativa do carbono estocado na biomassa das árvores foi obtida pela multiplicação das estimativas de biomassa obtidas pelo fator 0,5 , considerando-se que a biomassa seca contém aproximadamente 50\% de carbono (FUKUDA et al., 2003; SOARES e OLIVEIRA, 2002; FANG et al., 2001). Em seguida, o estoque de carbono foi extrapolado para toneladas por hectare.

\section{RESULTADOS}

\subsection{Estimativa dos parâmetros quantitativos}

A estrutura diamétrica da capoeira foi elaborada com base em seis classes de diâmetro, com um intervalo de classe de $5 \mathrm{~cm}$. Foram avaliados 901 indivíduos nas 10 parcelas avaliadas, que totalizaram $12,33 \mathrm{~m}^{2}$.ha ${ }^{-1}$ de área basal e 33,51 $\mathrm{m}^{3}$.ha-1 de volume de fuste (Tabela 3 ). 
Tabela 3 - Amplitude e variação dos parâmetros da comunidade da capoeira.

Table 3 - Amplitude and variation of community parameters in the capoeira.

\begin{tabular}{ccccc}
\hline Variáveis & Mínimo & Máximo & Média & Desvio padrão \\
\hline $\mathrm{N} / \mathrm{ha}$ & 1.020 & 2.440 & 1.802 & 403 \\
$\mathrm{AB}\left(\mathrm{m}^{2} \cdot \mathrm{ha}^{-1}\right)$ & 4,65 & 18,23 & 12,33 & 4,37 \\
$\mathrm{Vt}\left(\mathrm{m}^{3} \cdot \mathrm{ha}^{-1}\right)$ & 19,17 & 102,39 & 60,43 & 25,04 \\
$\mathrm{q}(\mathrm{cm})$ & 7,62 & 11,17 & 9,19 & 1,09 \\
$\bar{H}(\mathrm{~m})$ & 5,1 & 7,0 & 6,1 & 0,6 \\
\hline
\end{tabular}

Em que: $\mathrm{N} / \mathrm{ha}=$ número de indivíduos por hectare; $\mathrm{AB}=$ área basal por hectare; $\mathrm{Vt}=$ volume total com casca por hectare; q = diâmetro médio; e $\bar{H}=$ altura total média da árvore.

A classe com maior número de indivíduos foi a primeira (5-10 cm), que contribuiu com 78,25\% do total. As duas últimas classes (25-30 e 30-35 cm) apresentaram, cada uma, apenas $0,44 \%$ do número total de indivíduos (Figura 2).

\subsection{Quantificação da biomassa e do carbono estocado na capoeira}

Tomando-se como base os dados de densidade relativa e de valor de importância, foram selecionadas 10 espécies, com VI total de 58,9\%, para a determinação da densidade básica média da capoeira (Tabela 4).

A partir da densidade básica média ponderada da capoeira $\left(0,65 \mathrm{t} . \mathrm{m}^{-3}\right)$, obtiveram-se a estimativa de biomassa das árvores igual a 38,99 t.ha-1 e um estoque

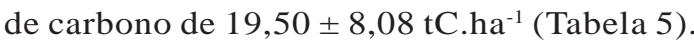

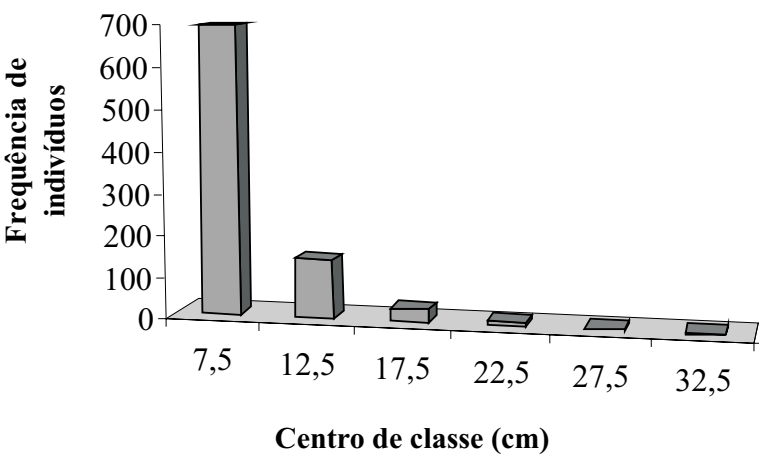

Figura 2 - Distribuição diamétrica considerando-se a frequência dos indivíduos amostrados na capoeira.

Figure 2 - Diametric distribution considering the frequency of individuals sampled in the capoeira.

Tabela 5 - Estimativas de biomassa das árvores com casca $\left(\right.$ t.ha $\left.{ }^{-1}\right)$ e carbono $\left(\right.$ t.ha $\left.^{-1}\right)$ da capoeira por parcela.

Table 5 - Estimates of trees biomass with bark (t.ha $\left.{ }^{-1}\right)$ and carbon $\left(\mathrm{t} \cdot \mathrm{ha}^{-1}\right)$ per plot of capoeira.

\begin{tabular}{ccc}
\hline Parcelas & Biomassa $\left(\mathrm{t} . \mathrm{ha}^{-1}\right)$ & $\mathrm{C}\left(\mathrm{t} . \mathrm{ha} \mathrm{A}^{-1}\right)$ \\
\hline 1 & 32,28 & 16,14 \\
2 & 66,06 & 33,03 \\
3 & 28,69 & 14,35 \\
4 & 41,29 & 20,64 \\
5 & 28,44 & 14,22 \\
6 & 12,37 & 6,18 \\
7 & 29,02 & 14,51 \\
8 & 57,02 & 28,51 \\
9 & 53,47 & 26,74 \\
10 & 41,26 & 20,63 \\
\hline Média \pm DP & $38,99 \pm 16,15$ & $19,50 \pm 8,08$ \\
\hline
\end{tabular}

Em que, DP = desvio-padrão.

Tabela4 - Densidade Relativa (DR), Valor de Importância (VI) e Densidade Básica da Madeira (DBM) das espécies selecionadas (maiores VI) na capoeira.

Table 4 - Relative Density (RD), Importance Value (IV) and Wood Basic Density (WBD) of the selected species in the capoeira (decreasing order of VI).

\begin{tabular}{|c|c|c|c|c|}
\hline Famílias & Espécies selecionadas & $\mathrm{DR}(\%)$ & VI(\%) & $\mathrm{DBM}\left(\mathrm{t} . \mathrm{m}^{-3}\right)$ \\
\hline Fabaceae & Piptadenia gonoacantha (Mart.) J. F. Macbr. & 12,03 & 15,73 & 0,75 \\
\hline Asteraceae & Vernonanthura diffusa (Less.) H. Rob. & 11,03 & 7,63 & 0,34 \\
\hline Melastomataceae & Miconia cinnamomifolia (DC.) Naudin & 6,77 & 7,63 & 0,73 \\
\hline Asteraceae & Piptocarpha macropoda (DC.) Baker & 7,52 & 6,97 & 0,48 \\
\hline Tiliaceae & Luehea grandiflora Mart. & 2,76 & 4,90 & 0,64 \\
\hline Fabaceae & Senna macranthera (DC. ex Collad.) H.S. Irwin e Barneby & 4,26 & 3,90 & 0,87 \\
\hline Monimiaceae & Siparuna guianensis Aubl. & 5,26 & 3,80 & 0,89 \\
\hline Bignoniaceae & Sparattosperma leucanthum (Vell.) K. Schum. & 4,26 & 3,73 & 0,57 \\
\hline Annonaceae & Xylopia sericea A. St.-Hil. & 2,51 & 2,35 & 0,53 \\
\hline \multirow[t]{2}{*}{ Meliaceae } & Trichilia pallida Sw. & 3,26 & 2,24 & 0,74 \\
\hline & & Total & 58,89 & \\
\hline
\end{tabular}




\section{DISCUSSÃO}

\subsection{Estimativa dos parâmetros quantitativos}

A estrutura diamétrica da capoeira (Figura 2) apresentou-se com tendência a um J-invertido, em que o maior número de indivíduos foi registrado nas menores classes de diâmetro, o que, segundo Schaaf et al. (2006), é um comportamento típico de florestas multiâneas.

A primeira classe de diâmetro (centro de classe de $7,5 \mathrm{~cm}$ ) apresentou 78,25\% do total de indivíduos da amostra, não sendo observadas lacunas nas classes de diâmetro. A alta concentração de indivíduos nas classes menores de diâmetro pode indicar uma comunidade estável e em regeneração (OLIVEIRA et al., 1989; OLIVEIRA-FILHO et al., 1996). No entanto, Machado et al. (2004) destacaram que a abundância de indivíduos de menores diâmetros pode estar associada a um regime de perturbações intensas e contínuas. Esse pode ser o caso da capoeira amostrada, visto o fragmento estar sujeito a efeitos de borda e à presença de gado bovino.

No que se refere aos parâmetros da comunidade vegetal (Quadro 3) e ao que estabelece a Resolução $n^{\circ} 392$ do CONAMA (2007), infere-se que a capoeira pode se enquadrar na classificação de Floresta Estacional Semidecidual em estádio secundário médio de regeneração. $\mathrm{O}$ fragmento apresenta altura média e DAP médio variando entre 5 a $12 \mathrm{~m}$ e 10 e $20 \mathrm{~cm}$, respectivamente. Além disso, mais da metade das espécies de maior VI (Piptadenia gonoacantha, Vernonanthura diffusa, Miconia cinnamomifolia, Luehea grandiflora, Senna macranthera, Xylopia sericea) se enquadram como espécies indicadoras desse estádio sucessional, conforme critérios da Resolução.

\subsection{Quantificação dos estoques de biomassa e carbono}

A estimativa de biomassa total da árvore registrada neste estudo foi menor do que o valor encontrado por Saldarriaga et al. (1988) que, em trabalho sobre o desenvolvimento sucessional na Região Amazônica, obtiveram valores iguais a 159 t.ha ${ }^{-1}$ e 116 t.ha ${ }^{-1}$ para a biomassa de árvores em áreas de floresta secundária com 40 e 60 anos, respectivamente. Essas áreas eram anteriormente usadas para agricultura e a quantificação de biomassa abrangeu todos os indivíduos amostrados, porém não fica claro se a casca também foi incluída nessa análise.

O contraste de valores deste estudo em relação aos de Saldarriaga et al. (1988) pode ser devido à ocorrência de distúrbios, como a presença de gado e a ação de efeitos de borda.

Avaliando estudos em que foi obtida a biomassa acima do solo no Bioma Mata Atlântica, observou-se que as estimativas de biomassa são expressivamente maiores do que as encontradas neste trabalho (Quadro 6).

Diversos fatores podem ter contribuído para a diferença de valores observada. Os métodos de amostragem usados foram distintos em relação aos deste estudo. Os trabalhos citados abrangeram desde o uso de transectos e parcelas circulares de diferentes raios (TIEPOLO et al., 2002) até o método da escalada para a coleta de dados (TANIZAKI-FONSECA, 2000), o que pode levar a diferentes valores. Além disso, para a estimativa da biomassa pelos estudos citados foram usadas diferentes equações, segundo o tipo florestal avaliado, o que também pode ter contribuído para as diferenças observadas. Algumas das equações apresentavam como variáveis independentes apenas o DAP ou o DAP e a altura, estando a densidade básica da madeira ausente na maioria dos casos.

As florestas abrangidas nesses estudos, apesar de também fazerem parte do Bioma Mata Atlântica, estavam localizadas nos Estados do Rio de Janeiro (TANIZAKI-FONSECA, 2000), de São Paulo(POMPÉIA, 1997 apud BURGER, 2005) e de Paraná (TIEPOLO et al., 2002). Dessa forma, é possível que características próprias de cada formação tenham contribuído para os diferentes estoques de biomassa em cada local.

Tabela 6-Estimativas de biomassa e carbono em florestas de Mata Atlântica com diferentes idades. Table 6 - Estimates of biomass and carbon in Atlantic Forest with different ages.

\begin{tabular}{|c|c|c|c|c|}
\hline Autor & Idade & Local & Biomassa (t.ha-1) & Carbono (tC.ha-1) \\
\hline Pompéia (1997) apud Burger (2005) & 25 anos & Cubatão (SP) & $186,4 / 64,6^{*}$ & - \\
\hline Tanizaki-Fonseca (2000) & 20 anos / 40 anos & Rio de Janeiro & $30 \pm 10 / 150 \pm 45$ & - \\
\hline Tiepolo et al. (2002) & FSJ / FSM & Paraná & - & $42,89 / 101,96$ \\
\hline
\end{tabular}

* Áreas de grande poluição atmosférica.

Em que: FSJ = floresta secundária jovem; e FSM = floresta secundária média.

Revista Árvore, Viçosa-MG, v.34, n.3, p.495-504, 2010 
Outro ponto relevante foi que algumas dessas formações florestais estavam localizadas em unidades de conservação (TIEPOLO et al., 2002) ou próximas a elas (POMPÉIA, 1997 apud BURGER, 2005) e, assim, sujeitas a práticas de preservação que podem favorecer o maior acúmulo de biomassa. Por fim, o tamanho e formato da capoeira no fragmento podem ter contribuído para um menor estoque de biomassa em relação aos demais estudos, os quais abrangeram formações florestais pouco estreitas e de maior área.

Os valores médios de biomassa total (38,99 t.ha $\left.{ }^{-1}\right)$ e da área basal registrados na capoeira $\left(12,33 \mathrm{~m}^{2} \cdot \mathrm{ha}^{-1}\right)$ não condizem com os esperados de uma floresta que apresenta histórico de cerca de 30 anos sem ser desmatada (SALDARRIAGA et al., 1988; TANIZAKIFONSECA, 2000). Além da constante presença do gado na capoeira, uma provável causa que possa estar contribuindo para os baixos valores encontrados é a forma estreita e comprida da área do fragmento no qual a capoeira se encontrava (Figura 1).

Laurance et al. (1997) demonstraram que a fragmentação leva a perdas significativas de biomassa, principalmente nas áreas com distâncias menores do que $100 \mathrm{~m}$ da borda do fragmento, nas quais os efeitos de borda são incisivos. Ranta et al. (1998) apontaram que fragmentos pequenos e de formato irregular apresentam poucas áreas em seu interior sem a influência do efeito de borda. Esses autores destacaram, também que, dependendo da largura do fragmento, todo ele pode estar sob o efeito de borda. Possivelmente esse é o caso da capoeira.

Levando em conta que a área do fragmento onde a capoeira se encontra apresenta, em sua parte mais larga, cerca de 165 m de largura, observa-se que toda essa área estaria sujeita aos efeitos de borda (alterações microclimáticas, distúrbios associados à excessiva passagem de vento e aumento da taxa de mortalidade de árvores, entre outros), o que pode ter contribuído para os baixos valores de biomassa total e área basal encontrados.

Laurance et al. (1998) destacaram que as alterações na dinâmica da floresta advindas da fragmentação florestal podem levar a uma expressiva perda de biomassa arbórea acima do solo e que esses efeitos são mais marcantes em fragmentos pequenos ( $<100$ ha) e de formato irregular. Isso pode estar associado à mortalidade de indivíduos e, até mesmo, à redução da riqueza de espécies durante e após o processo de fragmentação, o que é característico em fragmentos pequenos (TURNER et al., 1996).

Outro ponto a ser ressaltado é a relação do incremento de biomassa com o uso anterior da área. Gehring et al. (2005) apontaram que os impactos negativos do uso prolongado da terra no estoque de biomassa são mais evidentes na regeneração inicial e essas diferenças tendem a diminuir com o passar do tempo. A capoeira, por possuir apenas cerca de 30 anos e ter sido usada como pastagem, pode ainda estar sofrendo influência desse uso anterior.

A magnitude dessa influência, segundo Steininger (2000), está associada ao tempo de uso da área como pastagem e à intensidade desse uso. A utilização moderada tende a afetar pouco a taxa de acumulação de biomassa da regeneração no longo prazo, enquanto o uso muito intensivo inibe severamente essa taxa, em razão do pequeno número de sementes no solo. O mesmo caso foi observado por Ferguson et al. (2003), Moran et al. (1996) e Silver et al. (2000).

Estudos que promovem maior conhecimento acerca da quantidade de biomassa e carbono estocado em diferentes estádios sucessionais são de grande importância, pois fornecem subsídios para a elaboração de projetos de florestamento/reflorestamento no âmbito do Mecanismo de Desenvolvimento Limpo, que representa não só uma oportunidade para a geração de divisas para o Brasil, mas contribui para o seu desenvolvimento sustentável e a mitigação global da intensificação do efeito-estufa.

\section{CONCLUSÕES}

Diante do que foi exposto e pelos resultados alcançados conclui-se que

- A capoeira apresentou baixo estoque de carbono $(19,50$ t.ha-1 \pm 8,08 tC.ha-1) quando comparado com o de estudos realizados em fragmentos de idade semelhantes.

- O formato da parte do fragmento onde a capoeira se encontrava favoreceu a ação de efeitos de borda, o que pode ter contribuído com o estoque de carbono encontrado.

- O uso anterior da área pode ter afetado as taxas de acumulação de biomassa da regeneração da capoeira, levando à menor produção de biomassa no local. 
- As estimativas de estoque de carbono para a capoeira podem ser usadas como subsídios para a elaboração de projetos de florestamento/reflorestamento no âmbito do Mecanismo de Desenvolvimento Limpo.

\section{AGRADECIMENTOS}

À Sra. Terezinha Silva, por disponibilizar o sítio Bom Sucesso para a execução deste estudo, ao Márcio Assis e ao Laurindo Machado, pelo auxílio nas coletas de campo; e ao Conselho Nacional de Desenvolvimento Científico e Tecnológico-CNPq, pelo apoio financeiro.

\section{REFERÊNCIAS}

\section{ASSOCIAÇÃO BRASILEIRA DE NORMAS} TÉCNICAS - ABNT. NBR 11941: Madeira Determinação da densidade básica. 2003. 6p.

BRASIL. Ministério da Ciência e Tecnologia MCT. Protocolo de Quioto à convenção sobre mudança do clima. Brasília: 2001. 34p.

BURGER, D. M. Modelos alométricos para estimativa da fitomassa de Mata Atlântica na Serra do Mar, SP. 2005. 112f. Tese (Doutorado em Ciências) -

Universidade de São Paulo, São Paulo, 2005

CASTELLANI, D. C. et al. Produção de óleo essencial em catuaba (Trichilia catigua A. Juss) e negramina (Siparuna guianensis Aubl.) em função da época de colheita. Revista Brasileira de Plantas Medicinais, v.8, n.4, p.62-65, 2006.

\section{FUNDAÇÃO CENTRO TECNOLÓGICO DE MINAS GERAIS - CETEC. Determinação de equações volumétricas aplicáveis ao manejo sustentado de florestas nativas no estado de Minas Gerais e outras regiões do país. Belo Horizonte: SAT/CETEC, 1995. 295p.}

CHANG, M. Y. Seqüestro florestal de carbono no Brasil - Dimensões políticas socioeconômicas e ecológicas. In: SANQUETTA, C. R.; BALBINOT, R.; ZILLIOTTO, M. A. SIMPÓSIO LATINO AMERICANO SOBRE FIXAÇÃO DE CARBONO, 2., Fixação de carbono: atualidades, projetos e pesquisas. Curitiba: 2004. Anais...Curitiba: 2004. Parte 1. p.15-37.
CONSELHO NACIONAL DO MEIO AMBIENTE - CONAMA. Resolução $\mathbf{n}^{\circ}$ 392, de 25 de junho de 2007. Define vegetação primária e secundária de regeneração de Mata Atlântica no Estado de Minas Gerais. Disponível em: <http://www.mma.gov.br/port/ conama/legiabre.cfm?codlegi $=537>$. Acesso em: 16 maio 2008.

CORRÊA, G. F. Modelo de evolução e mineralogia da fração argila de solos do Planalto de Viçosa, MG. 1984. $87 \mathrm{f}$. Dissertação (Mestrado em Solos e Nutrição de Plantas) - Universidade Federal de Viçosa, Viçosa, MG, 1984.

FANG, J. et al. Changes in forest biomass carbon storage in China between 1949 and 1998.

Science, v.292, n.5.525, p.2.320-2.322, 2001.

FERGUSON, B. G. et al. Post-agricultural succession in El Petén, Guatemala. Conservation Biology, v.17, n.3, p.818-828, 2003.

FUKUDA, M.; IEHARA, T.; MATSUMOTO, M. Carbon stock estimates for sugi and hinoki forests in Japan. Forest ecology and management, v.184, n.1-3, p.1-16, 2003.

GEHRING, C. et al. Resilience of secondary forest regrowth after slash-and-burn agriculture in central Amazonia. Journal of Tropical Ecology, v.21, n.5, p.519-527, 2005.

HIGUCHI, N. et al. Dinâmica e balanço do carbono da vegetação primária da Amazônia Central. Revista Floresta, v.34, n.3, p.295-304, 2004.

IBAMA. Flora. Disponível em: <www.ibama.gov.br/flora/home.htm>. Acesso em: 26 fev. 2007.

LAURANCE, W. F. et al. Biomass collapse in Amazonian forest fragments. Science, v.278, n.340, p.1117-1118, 1997.

LAURANCE, W. F. et al. Rain forest fragmentation and the dynamics of Amazonian tree communities. Ecology, v.79, n.6, p.2032-2040, 1998.

LORENZI, H. Árvores brasileiras: manual de identificação e cultivo de plantas arbóreas nativas do Brasil. Nova Odessa: Plantarum, 2002a. 368p. v.1. 
LORENZI, H. Árvores brasileiras: manual de identificação e cultivo de plantas arbóreas nativas do Brasil. Nova Odessa: Plantarum, 2002b. 368p. v.2.

MACHADO, E. L. M. et al. Análise comparativa da estrutura e flora do compartimento arbóreo-arbustivo de um remanescente florestal na Fazenda Beira Lago, Lavras, MG. Revista Árvore, v.28, n.4, p.499-516, 2004.

MOBOT - Missouri Botanical Garden. w $^{3}$ TROPICOS. Disponível em: <http:// www.mobot.org/>. Acesso em: 14 fev. 2007.

MORAN, E. F. et al. Restoration of vegetation cover in the eastern Amazon. Ecological Economics, v.18, n.1, p.41-54, 1996.

OLIVEIRA, J. T. S.; HELLMEISTER, J. C.; TOMAZELLO FILHO, M. Variação do teor de umidade e da densidade básica na madeira de sete espécies de eucalipto. Revista Árvore, v.29, n.1, p.115-127, 2005.

OLIVEIRA, P. E. A. M.; RIBEIRO, J. F.; GONZALES, M. I. Estrutura e distribuição espacial de uma população de Kielmeyera coriacea Mart. de cerrados de Brasília.

Revista Brasileira de Botânica, v.12, n.1, p.39-47, 1989.

OLIVEIRA-FILHO, A. T.; CAMISÃO-NETO, A. A.; VOLPATO, M. M. L. Structure and dispersion of four tree populations in an area of montane semideciduous forest in southeastern Brazil. Biotropica, v.28, n.4, p.762-769, 1996.

PAULA, J. E.; IMAÑA-ENCINAS, J.; PEREIRA, B. A. S. Parâmetros volumétricos e da biomassa da mata ripária do córrego dos macacos. Cerne, v.2, n.2, p.91-105, 1996.

PINTO, S. I. C. Florística, estrutura e ciclagem de nutrientes em dois trechos de floresta estacional semidecidual na reserva florestal Mata do Paraíso, Viçosa-MG. 2005. 110f. Dissertação (Mestrado em Ciência Florestal) - Universidade Federal de Viçosa, Viçosa, MG, 2005.
RANTA, P. et al. The fragmented Atlantic rain forest of Brazil: size, shape and distribution of forest fragments. Biodiversity and Conservation, v.7, n.3, p.385-403, 1998.

REZENDE, S. B. Estudo da cronosequência em Viçosa - MG. 1971. 71f. Dissertação (Mestrado em Fitotecnia) - Universidade Federal de Viçosa, Viçosa, MG, 1971.

SALDARRIAGA, J. G. et al. Long-term chronosequence of forest succession in the upper Rio Negro of Colombia and Venezuela. Journal of Ecology, v.76, n 4, p.938958, 1988.

SCHAAF, L. B. et al. Alteração na estrutura diamétrica de uma floresta ombrófila mista no período entre 1979 e 2001. Revista Árvore, v.30, n.2, p.283-295, 2006.

SEGURA, M.; KANNINEN, M. Allometric models for tree volume and total aboveground biomass in a tropical humid forest in Costa Rica. Biotropica, v.37, n.1, p.2-8, 2005.

SILVA, A. F. et al. Composição florística e grupos ecológicos das espécies de um trecho de floresta semidecídua submontana da Fazenda São Geraldo, Viçosa-MG. Revista Árvore, v.27, n.3, p.311-319, 2003.

SILVER, W. L. et al. The potential for carbon sequestration through reforestation of abandoned tropical agricultural and pasture lands. Restoration Ecology, v.8, n.4, p.394-407, 2000.

SOARES JÚNIOR, F. J. Composição florística e estrutura de um fragmento de floresta estacional semidecidual na Fazenda Tico-Tico, Viçosa, MG. 2000. 68f. Dissertação (Mestrado em Botânica) - Universidade Federal de Viçosa, Viçosa, MG, 2000.

SOARES, C. P. B.; OLIVEIRA, M. L. R. Equações para estimar a quantidade de carbono na parte aérea de árvores de eucalipto em Viçosa, Minas Gerais. Revista Árvore, v. 26, n. 5, p. 533-539, 2002.

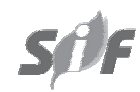

Revista Árvore, Viçosa-MG, v.34, n.3, p.495-504, 2010 
STEEGE, $H$. ter et al. Plant diversity in

Guyana: implications for a national protected areas strategy. Disponível em: <http://www.bio.uu.nl/ $\sim$ herba/Guyana/Plant_Diversity_Guyana/>. Acesso em: 22 maio 2007.

STEININGER, M. K. Secondary forest structure and biomass following short and extended land-use in central and southern Amazonia. Journal of Tropical Ecology, v.16, n.5, p.689-708, 2000.

TANIZAKI-FONSECA, K. Impacto do uso da terra no estoque $e$ fluxo de carbono na área de domínio da Mata Atlântica: estudo de caso, estado do Rio de Janeiro. 2000. 212f. Tese (Doutorado em Geociências) -

Universidade Federal Fluminense, Niterói, 2000
TIEPOLO, G.; CALMON, M.; FERETTI, A. R. Measuring and monitoring carbon stocks at the Guaraqueçaba climate action project, Paraná, Brazil. In: INTERNATIONAL SYMPOSIUM ON FOREST CARBON SEQUESTRATION AND MONITORING, 2002, Taipei, Taiwan. Anais... Taipei: Taiwan Forestry Research Institute, 2002. p.98-115.

TURNER, I. M. et al. A century of plant species loss from an isolated fragment of lowland tropical rain forest. Conservation Biology, v.10, n.4, p.1229-1244, 1996.

VELOSO, H. P.; FILHO, A. L. R. R.; LIMA, J. C. Classificação da vegetação brasileira, adaptada a um sistema universal. Rio de Janeiro: IBGE, 1991. 125p. 